\title{
Opportunistic Spectrum Aggregation for Cognitive Communications Under Collision Constraints
}

\author{
Haeyoung Lee ${ }^{1}$, Youngwook Ko ${ }^{2}$, Seiamak Vahid ${ }^{1}$, and Klaus Moessner ${ }^{1}$ \\ ${ }^{1}$ Centre for Communication Systems Research (CCSR), Department of Electronic Engineering, University of Surrey, U.K. \\ ${ }^{2}$ Institute of Electronics, Communications and Information Technology, Queen's University of Belfast, U.K. \\ Email: \{Haeyoung.Lee, S.Vahid, K.Moessner\}@ surrey.ac.uk ${ }^{1}$, Y.Ko@ qub.ac.uk ${ }^{2}$
}

\begin{abstract}
We consider a collision-sensitive secondary system that intends to opportunistically aggregate and utilize spectrum of a primary system to achieve higher data rates. In such opportunistic spectrum access, secondary transmission can collide with primary transmission. When the secondary system aggregates more channels for data transmission, more frequent collisions may occur, limiting the performance obtained by the opportunistic spectrum aggregation. In this context, dynamic spectrum aggregation problem is formulated to maximize the ergodic channel capacity under the constraint of collision tolerable level. To solve the problem, we develop the optimal spectrum aggregation approach, deriving closed-form expressions for the collision probability in terms of primary user traffic load, secondary user transmission interval, and the random number of sub-channels aggregated. Our results show that aggregating only a subset of sub-channels will be a better choice, depending on the ratio of collision sensitivity requirement to the primary user traffic.
\end{abstract}

Keywords-Spectrum aggregation, Collision probability, Opportunistic Spectrum Access

\section{INTRODUCTION}

By enabling the secondary users (SUs) to exploit the unoccupied spectrum of the primary users (PUs), the opportunistic spectrum access is regarded as a promising solution for resolving spectrum underutilization [1].

In the opportunistic spectrum access, since PUs have the priority over SUs in accessing the channels, PUs can access the channel a SU is using at any time. If such collision happens between PU and SU transmission, the SU has to vacate the channel immediately and move to a new one. While the SU communication has to be interrupted, packets must wait in the transmission buffer. The communication can be resumed when a connection is successfully established on a new channel. Such spectrum handoff naturally causes additional latency that affects SU performance in addition to causing short-term interference to PUs [2].

When a SU requires high data rates, the SU can use multiple channels simultaneously through spectrum aggregation [3]. However, the use of an aggregate channel comprising of multiple channels could incur frequent collisions. While improvement of the SU's performance could be expected by spectrum aggregation, frequent collisions would result in performance degradation of the SU. It is possible that the SU receives little benefit from spectrum aggregation. Thus, when the SU aggregates multiple channels, collision issues should be considered more carefully.
However, in literature, spectrum aggregation strategies of the secondary system proposed to date, only aim to improve the spectrum utilization without consideration of collision. In [4][5], the research focuses on how many channels should be aggregated, in order to accommodate more SUs and to improve data rates. In [6][7], while considering the hardware constraint for spectrum aggregation, aggregation algorithms with only the aim to achieve higher network throughputs have been proposed. Even though the collision probability of the SU [8] and spectrum allocation strategies for the secondary system considering the SU's collision probability have been investigated in [9][10][11], they only consider the single channel use by the SU. To the best of our knowledge, however, spectrum aggregation by secondary users considering the collision issue has not been covered in the literature.

In this paper, we consider a collision-sensitive secondary system opportunistically accessing multiple primary channels and aim at developing a new method for dynamic spectrum aggregation so that the ergodic capacity of an aggregate channel is maximized under the constraint that the collision probability is guaranteed to be smaller than some threshold value. For the aggregate channel use, the closed-form expression of the collision probability is derived. By analysing the impact of spectrum aggregation on the ergodic capacity and the collision probability, we address the challenge to maximize the ergodic capacity of the aggregate channel under the collision probability constraint. Moreover, a new optimization problem is formulated and by solving it, we intend to answer to a fundamental question, how many sub-channels should be activated for the spectrum aggregation.

The rest of this paper is organized as follows. Section II presents the system and channel models for primary and secondary system, describing the traffic pattern and the dynamic spectrum aggregation scheme. The spectrum aggregation problem to maximize the ergodic channel capacity considering the collision probability is formulated in Section III. The ergodic capacity and the collision probability are analysed in Section IV. Then, the optimal solution is addressed in Section V. After showing the numerical and simulation results in Section VI, our conclusions are summarized in Section VII.

\section{SyStem AND CHANNEL MODEL}

\section{A. System model}

We consider a secondary system coexisting with a primary system. The primary system communicates with the PUs 
through multiple licensed channels. The PUs, as the licensed users, have priority over the SUs in accessing the licensed (primary) channels. Thus, the secondary system opportunistically detects and aggregates idle primary channels, and allocates the aggregate channel (consisting of multiple primary channels) for the data transmission to the SU.

We assume that in the primary system, the packet arrival rate at each channel follows a Poisson process with a rate $\lambda_{p}$. The service time of the packets, $1 / \mu_{p}$, has the general distribution [11]. The packet arrival rate and service time at each channel are assumed to be independent and identically distributed (i.i.d.).

Suppose that in the secondary system, the base station (BS) is able to identify the availability of primary channels by spectrum sensing without errors. Particularly, every sensing period, the BS will identify a subset of primary channels unoccupied by PUs. For given subset of $N$ unoccupied channels, the BS sends the data to a SU during the data transmission interval. The data transmission interval of the secondary system is denoted by $T_{d}$, which is in general much longer than the sensing interval. $T_{d}$ is the maximum time interval over which a SU remains unaware of any changes in channel occupancy.

Consider a realistic case of the opportunistic spectrum access where PUs can return to the channels that are still used by the SU. In such case, collision can happen between primary and secondary transmissions. Such collision is inherent in realistic systems where no synchronization between the primary and secondary transmissions is assumed. To measure such collision, we define the collision probability, $P_{c}$, as the probability that the secondary transmission collides with the primary transmission and it can be given by [8]

$$
P_{c}=\lim _{T \rightarrow \infty} \frac{\text { the number of collided SU transmissions in }[0, T]}{\text { the number of SU transmissions in }[0, T]} .
$$

In the presence of certain collision, the secondary system can perform effective communication while $P_{c}$ remains below the pre-defined threshold of collision (denoted by $\xi_{t h}$ ). The collision threshold indicates the maximum collision probability tolerated by the secondary system. Notice that the value of $\xi_{t h}$ can influence the performance of the secondary system as well as that of the primary system. That is, until PUs are discovered from the time they collide with the SU, PUs can suffer from short-term interference. Thus, the quality of service of PUs could be also considered in the setting of $\xi_{t h}$.

\section{B. Channel model}

For given $N$ unoccupied primary channels, we propose that the BS of the secondary system aggregates only a subset of $n$ primary channels, properly selecting the value of $n(\leq N)$. Particularly, the BS randomly selects $n$ among $N$ channels every transmission interval $T_{d}$. That is, each one of the $N$ channels is equally likely to be selected and allocated at every transmission interval. Let the maximum transmission power be denoted by $P_{\max }$, and $P_{\max }$ is equally distributed among the $n$ randomly selected channels. Since the $n$ primary channels now comprise an aggregate channel, hereafter, the term 'primary channel' can be interchangeable with 'sub-channel (of an aggregate channel)'.
Suppose that available sub-channels between the BS and the SU are independent and Rayleigh flat fading. The channel coefficient of sub-channel $i$ (denoted by $h_{i}$, for all $i$ ) is complex Gaussian random variable with zero mean and variance $\sigma_{h_{i}}^{2}$, i.e., $h_{i} \sim \mathcal{C N}\left(0, \sigma_{h_{i}}^{2}\right)$. Such channel state information (CSI) is assumed to be perfectly known at the receiver. Then, the received signal to noise ratio (SNR) at sub-channel $i$ for $i \in\{1,2, \ldots, N\}$ can be represented as

$$
\rho_{i}=\frac{\left|h_{i}\right|^{2} P_{i}}{\sigma^{2}}=\frac{g_{i} P_{i}}{\sigma^{2}},
$$

where $g_{i}=\left|h_{i}\right|^{2}, \forall i$, is a Chi-square distributed random variable with $2 k$ degrees of freedom where $k$ denotes the number of receiver antennas along with multi-antenna techniques (e.g., maximal ratio combining) [12]. $P_{i}$ is the transmit power, i.e., $P_{i}=P_{\max } / n$ for equal power allocation. Notice in (2) that $\sigma^{2}$ is the variance of the complex-valued zero-mean additive white Gaussian noise (AWGN).

We can express the capacity of sub-channel $i$ of bandwidth $B$ as $C_{i}=B \log _{2}\left(1+\rho_{i}\right)$ [13]. Using (2), $C_{i}$ can be given by

$$
C_{i}\left(n, \frac{g_{i}}{\sigma^{2}}\right)=B \cdot \log _{2}\left(1+\frac{g_{i} \cdot P_{\max }}{n \cdot \sigma^{2}}\right) .
$$

From (3), the ergodic capacity for the selected sub-channel $i$ can be obtained by taking the expectation of (3) with respect to $g_{i} / \sigma^{2}$ which can be expressed as

$$
E\left[C_{i}\right]=\int_{0}^{\infty} B \cdot \log _{2}\left(1+P_{\max } \frac{x}{n}\right) p_{x}(x) \mathrm{d} x,
$$

where $E[\cdot]$ stands for the expectation operator and $p_{x}(x)$ is the pdf of $x=g_{i} / \sigma^{2}$, for all $i$, which can be given by

$$
p_{x}(x)=\frac{1}{2^{k / 2} \Gamma\left(\frac{k}{2}\right)} x^{\frac{k}{2}-1} e^{-\frac{x}{2}},
$$

where $\Gamma(\cdot)$ denotes the gamma function [13].

The capacity of the aggregate channel is upper bounded by the sum of the capacity of the $n$ active sub-channels, as the BS aggregates $n$ sub-channels to transmit the data. For the sake of the simplicity, let $B$ be normalized (i.e., $B=1$ ). Due to the proposed aggregation rule above, each of the $N$ sub-channels has the same probability to be randomly selected, according to the uniform distribution. The ergodic capacity, $C_{\text {total }}$, of the aggregate channel is calculated, weighting the ergodic capacity in (4) by its own aggregation probability and adding them all. Thus, $C_{\text {total }}$ can be expressed as

$$
C_{\text {total }}=\frac{n}{N} \sum_{i=1}^{N} E\left[\log _{2}\left(1+\frac{g_{i} \cdot P_{\max }}{n \cdot \sigma^{2}}\right)\right] .
$$

\section{PROBLEM FORMULATION}

We study the problem of the spectrum aggregation, properly selecting only a subset of $n$ among $N$ total idle subchannels for the secondary transmission. This is to maximize the achievable data rate $C_{\text {total }}$ while satisfying a collision probability requirement. Using (5), this problem can be posed as

$$
\begin{gathered}
\max C_{\text {total }}=\max \frac{n}{N} \sum_{i=1}^{N} E\left[\log _{2}\left(1+\frac{g_{i} \cdot P_{\max }}{n \cdot \sigma^{2}}\right)\right], \\
\text { subject to } P_{c, n} \leq \xi_{t h} .
\end{gathered}
$$


where $P_{c, n}$ denotes the probability that secondary transmission exploiting the $n$ sub-channels collides with the primary transmission.

Notice that collision by the secondary transmission on the $n$ sub-channels includes all the events of collision across all possible subsets of the $n$ sub-channels. Thus, for a given $n$, $P_{c, n}$ can be derived, utilising the collision probability of an individual sub-channel as follows:

$$
\begin{aligned}
P_{c, n} & =\mathrm{P}\{\text { Collision in any of } n \text { channels }\} \\
& =1-\mathrm{P}\{\text { No collision in all } n \text { channels }\} .
\end{aligned}
$$

To solve the problems (6)-(8), we raise and address a fundamental question, "how many sub-channels must be aggregated for higher data rate under the collision requirement?" To that end, we first start by analysing the impact of the number of aggregate sub-channels on the performance, followed by the optimal solution using analytical results.

\section{ANALYSIS ON ERGODIC CAPACITY AND COLLISION PROBABILITY}

\section{A. Impact of the channel aggregation on $C_{\text {total }}$}

Since the log function is a concave function, by using Jensen's inequality [13], the ergodic capacity of the aggregate channel composed of the $n$ sub-channels for a given $N$ can be obtained by

$$
\begin{aligned}
C_{\text {total }} & =\frac{n}{N} \sum_{i=1}^{N} E\left[\log _{2}\left(1+\frac{g_{i} \cdot P_{\text {max }}}{n \cdot \sigma^{2}}\right)\right] \\
& \leq \frac{n}{N} \sum_{i=1}^{N} \log _{2}\left(1+\frac{\gamma_{i}}{n}\right),
\end{aligned}
$$

where $\gamma_{i}$ denotes the average received SNR level with the maximum transmit power on sub-channel $i$, i.e., $\gamma_{i}=$ $E\left[g_{i}\right] \cdot P_{\max } / \sigma^{2}$.

Let us figure out how the ergodic capacity behaves with $n$, the number of sub-channels used for aggregation. For this, the first and the second derivatives of the ergodic capacity with respect to $n$ are computed as follows

$$
\begin{gathered}
\frac{\partial C_{\text {total }}}{\partial n}=\frac{1}{N(\log 2)} \sum_{i=1}^{N}\left[\log \left(1+\frac{\gamma_{i}}{n}\right)-\frac{\gamma_{i}}{n+\gamma_{i}}\right] \\
\frac{\partial^{2} C_{\text {total }}}{\partial^{2} n}=\frac{1}{N(\log 2)} \sum_{i=1}^{N} \frac{-\gamma_{i}^{2}}{n\left(\gamma_{i}+n\right)^{2}}
\end{gathered}
$$

Consider the homogeneous channel setup where $\gamma_{i}=\gamma, \forall i$. Noticing the fact that $\partial^{2} C_{\text {total }} / \partial^{2} n$ in (11) is not positive for all possible $n, C_{\text {total }}$ is a concave function of $n$. The first derivative function is always positive (i.e., $\partial C_{\text {total }} / \partial n>0$ ). This reveals that the ergodic capacity monotonically increases with $n$. Therefore, for a given finite $N$, more $n$, higher $C_{\text {total }}$ is obtained.

\section{B. Impact of the channel aggregation on collision probability}

Let us consider the homogeneous case when the PU traffic intensity on sub-channel $i, \forall i$ are i.i.d. The collision probability of each sub-channel is equally likely being $P_{c}$ in (1). Using this, (8) can be further simplified to

$$
P_{c, n}=1-\prod_{i=1}^{n}\left(1-P_{c}\right)=1-\left(1-P_{c}\right)^{n} .
$$

It can be shown in (12) that the collision probability, $P_{c, n}$ can be derived as an increasing function of the number of subchannels, $n$, once the collision probability by a single subchannel use, $P_{c}$ is given.

To further analysis on $P_{c, n}$, we now calculate $P_{c}$. The collision happens only if PUs reappear on the sub-channels on which the SU is still transmitting. As shown in [8], the collision probability of a single sub-channel due to asynchronous transmission between primary and secondary transmission, $P_{c}$, is equivalent to the probability that at least one PU's packet arrives during a SU's transmission period $T_{d}$. As PU's packets arrive according to a Poisson process, $P_{c}$, can be given by

$$
P_{c}=1-\exp \left(-\lambda_{P} \cdot T_{d}\right)
$$

From (12) and (13), $P_{c, n}$ can be given, for given $n$ aggregated sub-channels, by

$$
P_{c, n}=1-\left(1-P_{c}\right)^{n}=1-\exp \left(-\lambda_{P} \cdot T_{d} \cdot n\right) .
$$

In (14), $P_{c, n}$ indicates the probability that at least one primary packet arrives during a secondary transmission period in $n$ subchannels. It can be seen from (14) that $P_{c, n}$ increases with the product of $\lambda_{P}$ and $T_{d}$, for given $n$ aggregated sub-channels. For given $\lambda_{P}$ and $T_{d}$, also, it can be shown from (14) that $P_{c, n}$ increases significantly with $n$.

\section{OPTIMAL SOLUTION}

We consider the collision-sensitive secondary system where it is desired to provide a realistic optimal solution that maximizes the ergodic capacity $C_{\text {total }}$, while simultaneously limiting the collision probability $P_{c, n}$ below the desired level. For this, we solve the maximization problem described in (6) and (7).

As per $C_{\text {total }}$ and $P_{c, n}$, the analytical results in Section IV presented that the both are functions of the number $n$ of active sub-channels used for aggregation. Particularly, notice the fact that the monotonicity of $C_{\text {total }}$ with $n$ presented in Section IV. Then, it can be stated that, for a given collision probability threshold $\xi_{t h}$, the optimal value of $n(\leq N)$ maximizing $C_{\text {total }}$ may exist and can be found as the largest among possible integer values satisfying the collision probability requirement $\left(P_{c, n} \leq \xi_{t h}\right)$. The requirement (7) can be rewritten, using (12), as

$$
1-\left(1-P_{c}\right)^{n} \leq \xi_{t h}
$$

Taking the logarithm of both sides in (15), the necessary condition on being possible candidate $n$ s for the optimum can be obtained for a given $\xi_{t h}$ as

$$
n \leq\left\lfloor\frac{\log \left(1-\xi_{t h}\right)}{\log \left(1-P_{c}\right)}\right\rfloor
$$


where $\lfloor x\rfloor$ denotes the largest integer not larger than $x$, and $P_{c}$ denotes the collision probability being (13). Here, note that $n$ is the number of active sub-channels for aggregation, and thus the valid range of $n$ in (16) must be given to $1 \leq n \leq N$.

Considering the requirement in (15), the optimum $n^{*}$ must be the largest integer satisfying the inequality of (16). Thus, $n^{*}$ subject to $P_{c, n} \leq \xi_{t h}$ can be expressed using (16).

$$
n^{*}=\left[\begin{array}{cl}
\left\lfloor\frac{\log \left(1-\xi_{t h}\right)}{\log \left(1-P_{c}\right)}\right\rfloor, & \text { if } 1 \leq\left\lfloor\frac{\log \left(1-\xi_{t h}\right)}{\log \left(1-P_{c}\right)}\right\rfloor \leq N \\
N, & \text { elseif }\left\lfloor\frac{\log \left(1-\xi_{t h}\right)}{\log \left(1-P_{c}\right)}\right\rfloor>N \\
0, & \text { otherwise }
\end{array}\right] .
$$

It can be shown from (17) that $n^{*}$ relies on both $P_{c}$ and $\xi_{\text {th }}$ for a given $N$.

To further specify $n^{*}, P_{c}$ in (13) are now taken into account, solving (17). To that end, we need to derive the expression for $n^{*}$ with respect to the primary packet arrival rate $\lambda_{p}$ and the SU transmission interval $T_{d}$. For simplicity in analysis, hereinafter, we consider only when all the candidates satisfying (16) are below $N$, i.e., $1 \leq\lfloor x\rfloor \leq N$, for a given $N$. This leads us to consider only the first inequality condition in (17). Since in the other conditions in (17) the corresponding optimal solutions can be provided straightforwardly, being beyond our interests.

Inserting (13) into (17), therefore, $n^{*}$ can be found as follows

$$
n^{*}=\left\lfloor\frac{\log \left(1-\xi_{t h}\right)}{-\lambda_{p} \cdot T_{d}}\right\rfloor .
$$

In (18), it is observed that for a given collision requirement $\xi_{t h}$, the optimal number of sub-channel for aggregation should be chosen inversely with a product of $\lambda_{p} \cdot T_{d}$. Particularly, it can be found from (18) that lower $\lambda_{p}$ (or smaller $T_{d}$ ), more $n^{*}$ can be. This reveals that as the product $\lambda_{p} \cdot T_{d}$ decreases, the optimum number $n^{*}$ of the aggregate sub-channels are allowed to increase. Intuitively, at the low rate of primary packets arrival in a given secondary transmission interval, resulting large active sub-channels for the aggregation leads to the increase in the ergodic capacity of an aggregated channel. Moreover, it is worth mentioning that when $\xi_{t h}$ increases (towards 1) for a given $\lambda_{p} \cdot T_{d}, n^{*}$ in (18) also does. This indicates that large sub-channels can be exploited for the aggregation for a collision-tolerated secondary system with high $\xi_{t h}$. Similarly, as $\xi_{t h}$ decreases for the collision-sensitive system, $n^{*}$ should exponentially decreases for a given $\lambda_{p} \cdot T_{d}$.

For example, let us consider the homogeneous channel environments where $\gamma_{i}=\gamma, \forall i$. Using (9) and (18), the maximum ergodic capacity can be derived as follows

$$
C_{\text {total }}=\left\lfloor\frac{\log \left(1-\xi_{\text {th }}\right)}{-\lambda_{p} \cdot T_{d}}\right\rfloor \log _{2}\left(1+\frac{\gamma}{\left\lfloor\frac{\log \left(1-\xi_{t h}\right)}{-\lambda_{p} \cdot T_{d}}\right\rfloor}\right),
$$

where recall that $\lfloor$.$\rfloor equals n^{*}$ in (18). It can be shown from (19) that $C_{\text {total }}$ increases monotonically whatever $n^{*}$ grows, resulting from either 1) low $\lambda_{p} \cdot T_{d}$ or 2) high $\xi_{t h}(\leq 1)$. It is also worth mentioning that for a given $\xi_{t h}, C_{\text {total }}$ can remain at a certain minimum desired level, adjusting $T_{d}$ to the variations of $\lambda_{p}$. That is, when PU packet arrives more frequently (i.e., with large $\lambda_{p}$ ), it is desired to reduce $T_{d}$,
TABLE I. SIMULATION PARAMETERS

\begin{tabular}{|l|c|}
\hline Parameter & Value \\
\hline \hline The number of total sub-channels & 30 \\
\hline PU packet arrival rate, $\lambda_{p}$ & {$[0.02-0.6] / \mathrm{sec}$} \\
\hline PU packet service time, $1 / \mu_{p}$ & $1 \mathrm{sec}$ \\
\hline SU Transmission interval, $T_{d}$ & $10 \mathrm{~ms}$ \\
\hline Total transmit power, $P_{\max }$ & 1 (Equal power allocation) \\
\hline Average SNR, $E[g] / \sigma$ & $20 \mathrm{~dB}$ (Rayleigh fading) \\
\hline Collision probability threshold, $\xi_{t h}$ & 0.005 \\
\hline Simulation time & $10^{5} T_{d}$ \\
\hline
\end{tabular}

enabling more frequent spectrum sensing. This is in order to accurately detect the PU traffic arrival, leading $C_{\text {total }}$ to the desired level.

\section{Simulation RESUlts}

In this section, we present simulation results to evaluate the formulated collision probabilities and the proposed optimal aggregation algorithm's performance.

Simulations consist in a sequence of events, arrival or leave of primary packets at each sub-channel and secondary transmission using multiple sub-channels. We consider a total of 30 primary channels. Based on channel status information, a BS will randomly choose $n$ sub-channels considering a given collision probability threshold and communicate with the SU. The frequency of collision events are counted and the simulation results are averaged for the obtained results during $10^{5} T_{d}$. The parameters used for simulation are described in Table I.

Firstly, we validate our formulated collision probability that a SU experiences by using multiple sub-channels, described in Section IV. Fig. 1 presents the collision probabilities $P_{c, n}$ formulated in (14) with respect to various $\lambda_{p}$. For given $n \in$ $\{1,5,10\}, \lambda_{p}$ varies from 0.1 to 0.6 per sec and $1 / \mu_{p}$ is set to 1 sec. Fig. 1 shows that $P_{c, n}$ increases with $n$ and $\lambda_{p}$ for a given $T_{d}$ and that for different values of $n$, the simulation results closely follow and thus validate the numerical analysis $P_{c, n}$ calculated by (14).

Secondly, the performance of the optimal spectrum aggregation method considering the collision constraint is evaluated by simulation. We compare the performance of the optimal aggregation scheme with three fixed number of sub-channels

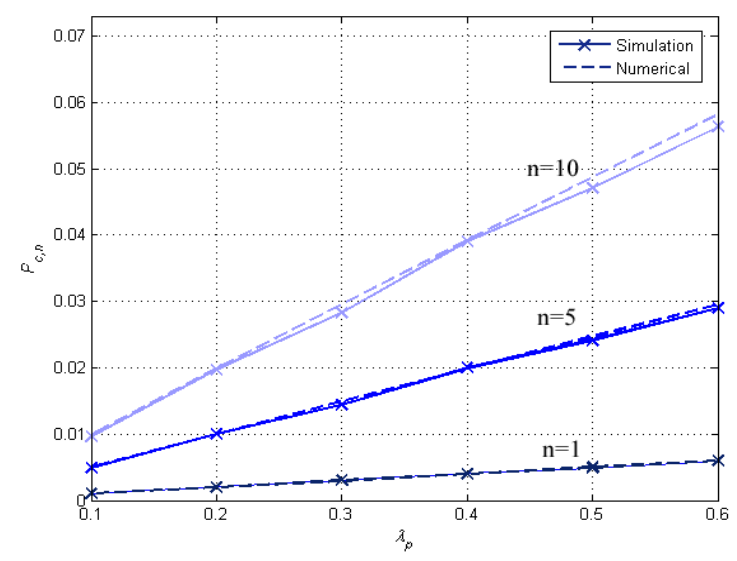

Fig. 1. Comparison of collision probabilities $P_{c, n}$ obtained from simulation and numerical analysis for various $\lambda_{p}$ 


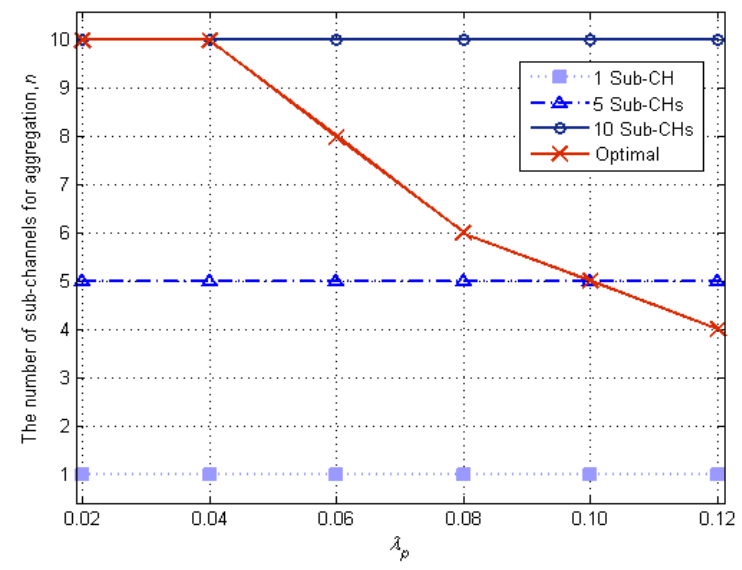

(a) The number of sub-channels for aggregation

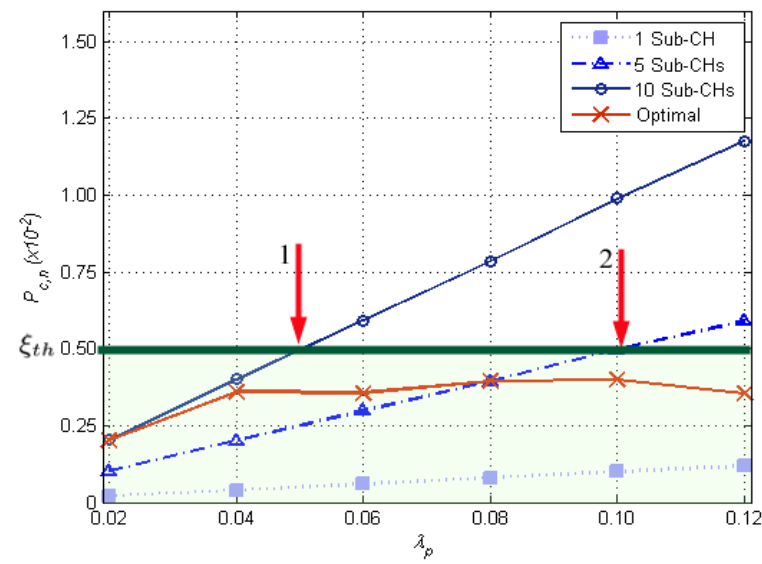

(b) Collision probability of an aggregate channel

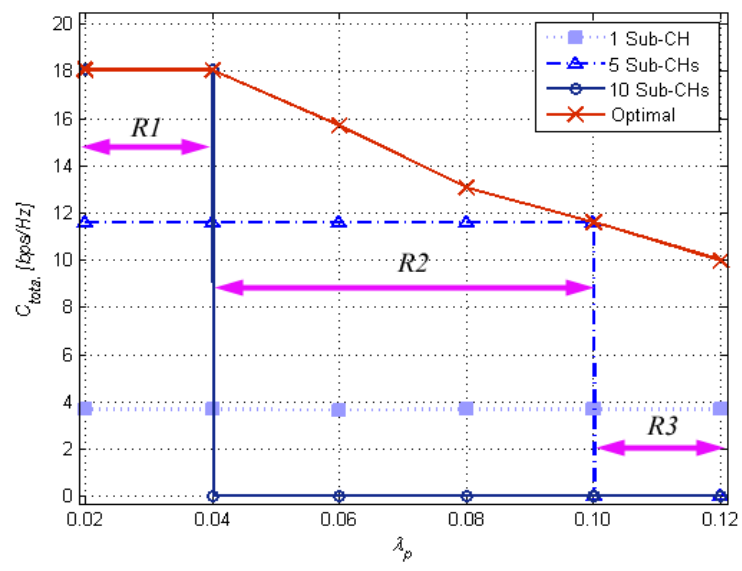

(C) Ergodic capacity of an aggregate channel

Fig. 2. Comparison of the proposed aggregation scheme with aggregation of the fixed number of sub-channels

aggregation schemes (i.e., 1, 5, and 10 sub-channels) as shown in Fig. 2. In Fig. 2(a), it is shown that whilst the three fixed aggregation schemes select a given fixed number of sub-channels, the optimal algorithm selects $n$ number of subchannels adaptively by (18) for given $\lambda_{p}, T_{d}$ and $\xi_{t h}$. In Fig. 2(b), the collision probability $P_{c, n}$ for four schemes is depicted for varying $\lambda_{p}$. Whilst $\xi_{t h}$ is set to 0.005 in this scenario, the valid scheme should generate $P_{c, n}$ lower than $\xi_{t h}$ for a given $\lambda_{p}$ and $T_{d}$. In the case of the " 1 Sub-CH" allocation scheme, its $P_{c, n}$ keeps lower value than $\xi_{t h}$ for various $\lambda_{p}$. However, for the "10 Sub-CHs" and "5 Sub-CHs" aggregation schemes, their $P_{c, n}$ exceeds $\xi_{t h}$ at the points of arrow marked by 1 and 2 , respectively. Since we consider the discrete range of $\lambda_{p}$ (from 0.02 to 0.12 with an interval of 0.02 ), under the collision probability constraint, the "10 Sub-CHs" aggregation scheme only remains valid for $\lambda_{p}=[0.02-0.04]$ and the "5 Sub-CHs" aggregation scheme remains valid only for $\lambda_{p}=[0.02-0.10]$. In the case of the optimal aggregation scheme, the $P_{c, n}$ for all $\lambda_{p}$ is kept under the $\xi_{t h}$ while it changes the number of subchannels for aggregation. Fig. 2(c) shows the ergodic capacity of the aggregate channel, $C_{\text {total }}$. For region 1 (marked by $\mathrm{R} 1)$ of $\lambda_{p}=[0.02-0.04]$, the "10 Sub-CHs" aggregation scheme achieves the highest $C_{\text {total }}$ among the fixed number of sub-channel aggregation schemes. Due to the $P_{c, n}$ larger than $\xi_{t h}$, for the region of $\lambda_{p}$ larger than 0.04 , the "10 SubCHs" aggregation scheme is no longer valid. The "5 SubCHs" aggregation scheme shows better $C_{\text {total }}$ than the "1 Sub-CH" allocation scheme for region 2 (marked by R2) of $\lambda_{p}=(0.04-0.10]$. However, for $\lambda_{p}$ larger than 0.10 , its $P_{c, n}$ becomes larger than $\xi_{t h}$. In region 3 (marked by R3) of $\lambda_{p}=(0.10-0.12]$, only the "1 Sub-CH" allocation scheme can be utilized. While the aggregation algorithms using a fixed number of sub-channel can be utilized within the limited range of $\lambda_{p}$, the optimal algorithm shows the highest $C_{\text {total }}$ among four schemes considered for all regions, R1, R2 and R3.

\section{CONCLUSION}

We have considered the dynamic spectrum aggregation for the collision-sensitive secondary system so that the ergodic capacity is maximized under the collision probability constraint. It was found that the ergodic capacity by the spectrum aggregation increases with the number of sub-channels activated for aggregation. The collision probability of the aggregate channel was analysed as the function of the PU packet arrival rate, SU transmission interval, and the number of subchannels activated for the spectrum aggregation. Our analytical and simulation results have clearly shown that the number of sub-channels activated for the spectrum aggregation must exponentially decrease in the highly collision-sensitive use case when decreasing the collision probability threshold. Also, it was found that, for a given collision probability threshold, lower PU packet arrival rate and shorter SU transmission interval can allow a SU to aggregate more sub-channels leading to higher data rates. Based on theses findings, the optimal spectrum aggregation strategy has been proposed to achieve the highest ergodic capacity under the given collision probability constraint. Through adaptive selection of number of subchannels, the proposed optimal scheme was shown to outperform the conventional spectrum aggregation the fixed number of sub-channels. Collisions between primary and secondary transmission can also occur due to noisy estimation of idle channels. Analysis of the impacts of such collisions will be investigated as part of our future work.

\section{ACKNOWLEDGMENT}

This work is performed as part of ACROPOLIS (www.ictacropolis.eu) projects supported by the European Communitys 
Seventh Framework Program (FP7) and EPSRC/DST IndiaUK Advanced Technology Centre (IU-ATC) programme.

\section{REFERENCES}

[1] Q. Zhao and B. Sadler, "A survey of dynamic spectrum access," IEEE Signal Process. Mag., vol. 24, no. 3, pp. 79-89, May 2007.

[2] I. Christian, S. Moh, I. Chung, and J. Lee, "Spectrum mobility in cognitive radio networks," IEEE Commun. Mag., vol. 50, no. 6, pp. 114-121, June 2012.

[3] H. Lee, S. Vahid, and K. Moessner, "A survey of radio resource management for spectrum aggregation in lte-advanced," IEEE Commun. Surveys Tuts., vol. PP, no. 99, pp. 1-16, Nov. 2013.

[4] J. Lee and J. So, "Analysis of cognitive radio networks with channel aggregation," in Proc. IEEE WCNC, Sydney, Australia, April 2010, pp. $1-6$.

[5] L. Jiao, F. Li, and V. Pla, "Dynamic channel aggregation strategies in cognitive radio networks with spectrum adaptation," in Proc. IEEE GLOBECOM, Houston, TX, US, Dec. 2011, pp. 1-6.

[6] J. Lin, L. Shen, N. Bao, B. Su, Z. Deng, and D. Wang, "Channel characteristic aware spectrum aggregation algorithm in cognitive radio networks," in Proc. IEEE LCN, Bonn, Germany, Oct. 2011, pp. 634639.

[7] D. Chen, Q. Zhang, and W. Jia, "Aggregation aware spectrum assignment in cognitive ad-hoc networks," in Proc. 3rd Int. Conf. CROWNCOM, Singapore, May 2008, pp. 1-6.

[8] M. d'Utra da Costa and P. Cardieri, "Collision probabilities for dynamic spectrum access with cognitive radios," in Proc. 2009 SBMO/IEEE MTT-S Int'l Microwave and Optoelectronics Conf. (IMOC), Belem, Brazil, Nov. 2009, pp. 272-276.

[9] X. Li, Q. Zhao, X. Guan, and L. Tong, "Optimal cognitive access of markovian channels under tight collision constraints," IEEE J. Sel. Areas Commun., vol. 29, no. 4, pp. 746-756, April 2011.

[10] Y. L. Che, R. Zhang, and Y. Gong, "On design of opportunistic spectrum access in the presence of reactive primary users," IEEE Trans. Commun., vol. 61, no. 7, pp. 2678-2691, July 2013.

[11] Q. Xiao, Y. Li, M. Zhao, S. Zhou, and J. Wang, "Opportunistic channel selection approach under collision probability constraint in cognitive radio systems," Computer Communications, vol. 32, no. 18, pp. 1914 - 1922, 2009. [Online]. Available: http://www.sciencedirect.com/science/article/pii/S0140366409001753

[12] Y. Ko and K. Moessner, "Maximum outage capacity in dense indoor femtocell networks with joint energy and spectrum utilization," IEEE Trans. Wireless Commun., vol. 11, no. 12, pp. 4416-4425, Dec. 2012.

[13] J. G. Proakis and M. Salehi, Digital Communications 5th Edition. McGraw Hill, 2007. 